A N N A L E S Annales de Bretagne et des Pays de l'Ouest

\title{
Les nouveaux patrimoines en Pays de la Loire
}

\section{Charles-Tanguy Le Roux}

\section{OpenEdition}

\section{Journals}

Édition électronique

URL : http://journals.openedition.org/abpo/2822

DOI : $10.4000 / a b p o .2822$

ISBN : 978-2-7535-3513-8

ISSN : 2108-6443

\section{Éditeur}

Presses universitaires de Rennes

Édition imprimée

Date de publication : 30 juin 2014

ISBN : 978-2-7535-3511-4

ISSN : 0399-0826

Référence électronique

Charles-Tanguy Le Roux, "Les nouveaux patrimoines en Pays de la Loire ", Annales de Bretagne et des Pays de l'Ouest [En ligne], 121-2 | 2014, mis en ligne le 30 juin 2014, consulté le 23 septembre 2020. URL : http://journals.openedition.org/abpo/2822 ; DOI : https://doi.org/10.4000/abpo.2822 
Les trois derniers articles sont le résultat d'une journée de séminaire organisée en mai 2011. Au contraire des deux autres ensembles, ils ne portent pas sur un thème défini et leur seul point commun tient au fait qu'ils ont été écrits par des chercheurs étrangers, spécialistes ou non de la matière bretonne. Sont ainsi traitées l'origine des cantiques vannetais du XVII ${ }^{\mathrm{e}}$ siècle - à travers l'analyse des rimes internes et de l'influence des modèles français - par Anders Richardt Jørgensen (p. 203-231) et l'image des langues minoritaires à travers les médias irlandais, maoris et bretons par Ruth Lysaght (p. 233-252). Un dernier article théorique de Vittorio Dell'Aquila et Gabriele Iannàccaro porte quant à lui sur les usages cartographiques au service de la géolinguistique (p. 253-286). Ces travaux rappellent qu'une part importante des recherches sur les sources bretonnes se fait en dehors de France, ce qui ne peut qu'inciter à la multiplication des rapprochements avec les réseaux internationaux dans le domaine des études celtiques.

Éva GUILLOREL

Morice, Jean-René, SAUPIN Guy, VERDIER, Nadine (dir.), Les nouveaux patrimoines en Pays de la Loire, Rennes, PUR, coll. " Art et société ", 2013, 756 p.

Publié avec le soutien de la Région Pays de la Loire, ce fort (et beau) volume est le fruit du travail de plus de quarante auteurs (relevant de huit laboratoires répartis dans trois universités), durant trois ans et sur un projet (NEOPAT) lui-même retenu par ladite région dans le cadre d'un appel à projets de recherche. Sans être une " commande " à proprement parler, il est clair que l'ouvrage répond, dans le domaine patrimonial, à un légitime souhait de lisibilité générale de leur région, ressenti par ses élus. On le sait, l'entité territoriale des Pays de la Loire est récente et a été dessinée assez artificiellement à partir de quatre anciennes provinces - Maine et Anjou, mais aussi Bretagne et Poitou. L'émergence actuelle de cette " conscience régionale " serait à comparer à celle qui s'est petit à petit forgée en plus de deux siècles autour de certains départements français, aux territoires parfois tout aussi hétéroclites. Il serait vain de rechercher ici un "traité " de patrimoine ligérien. Le but, comme exposé par le Président de Région dans sa préface est à la fois de dresser un "état " de ces patrimoines et d'étudier "leur part dans la construction des identités".

Dans leur présentation introductive (au style malheureusement parfois assez alambiqué), les directeurs de l'ouvrage présentent tout d'abord la notion de "nouveaux patrimoines " dans le cadre d'un domaine dont «l'expansion constante en fait un concept sans rivages " et où " des éléments du passé, jusqu'ici ignorés ou peu considérés, retiennent désormais l'attention ». Ils exposent ensuite les " trois grands objectifs scientifiques " de leur projet : analyser les conditions d'émergence des nouveaux objets patrimoniaux, établir un bilan du jeu des différents acteurs concernés, montrer comment ces démarches interviennent dans la construction des territoires. Qu'on ne s'attende pas à trouver ici un " traité " présentant les différents éléments de patrimoine pris en compte et qu'on ne s'étonne pas non plus des abondantes références faites aux structures et procédures techno-administratives (quitte à ce que les auteurs relèvent ici ou là la lourdeur du " millefeuilles "). Cependant, une grande latitude semble avoir été laissée aux contributeurs; selon leur sensibilité et la nature du sujet, l'exposé liminaire de celui-ci peut donc prendre des ampleurs ou des formes très variables.

Le cœur de l'ouvrage s'organise en trois "parties ", d'ailleurs très inégales : les regards de la presse régionale (en l'occurrence la revue trimestrielle régionale 
303 tout au long de ses trente ans d'existence et le grand quotidien interrégional Ouest-France pour la période 2010-2012) et " les enjeux des nouveaux patrimoines " (regroupant des réflexions transversales et une conclusion générale) encadrent une volumineuse " identification des nouveaux patrimoines " elle-même divisée en cinq chapitres; le fil conducteur des deux premiers peut se rattacher à ce que l'on appelait naguère la "géographie physique ", les suivants ressortant plus clairement de la " géographie humaine " (mais avec bien des nuances et approximations).

Les domaines de l'eau (fluviale et maritime) sont abordés en premier par onze articles. Le fleuve éponyme de la région y tient bien sûr la place d'honneur mais d'autres entités sont abordées (le canal de Nantes à Brest, la Mayenne). Navigation et batellerie sont privilégiées malgré un article sur les moulins et usines hydrauliques (thème qui réapparaîtra à propos du patrimoine industriel). Mais ces contributions restent indépendantes, ce qui occulte une constante dans l'appropriation du milieu fluvial, à savoir les antagonismes entre usages statiques et dynamiques de l'eau - entre pêcheurs et meuniers d'une part, bateliers et marchands d'autre part pour caricaturer. Quant à l'eau salée, elle est abordée par ce qu'elle a de plus emblématique dans la région, les salines de Guérande (les seules de France dont l'exploitation traditionnelle n'ait jamais cessé) avant de passer à une néo-valorisation du littoral vue à travers ces deux stations balnéaires si différentes dans leur histoire et leur actualité que sont Saint-Gilles-Croix-de-Vie et La Baule, cette dernière étant présentée à la faveur d'une étude sur le patrimoine urbain de villes - anciennes et fluviales celles-là - que sont Nantes, Angers et Saumur.

Le relief sert de dénominateur commun aux sept articles suivants, ce qui peut paraître osé dans une région qui porte il est vrai le modeste point culminant du Massif armoricain à ses confins nord mais qui reste fondamentalement un " plat pays " malgré des accidents géomorphologiques parfois spectaculaires et dont beaucoup méritent leur appropriation patrimoniale. Pour ce faire, les outils juridiques (traditionnels, nouveaux ou à inventer) sont passés en revue et évalués dans une étude spécifique. Notons aussi que le cadre régional strict de l'ouvrage devient parfois carcan dans les deux chapitres que nous venons de survoler; contentonsnous de deux exemples : a) comme chacun sait, la Loire prend sa source au mont Gerbier-de-Jonc dans le Vivarais et elle fut naviguée jusqu'en Nivernais, sans parler des " avalaisons" de charbon depuis les mines stéphanoises; b) comment comprendre le "Sillon de Bretagne " sans le situer dans le cadre du grand " cisaillement sud-armoricain " qui va de la Pointe du Raz au Limousin? Pour aider à leur compréhension, plusieurs cartes auraient mérité mieux qu'un blanc absolu au-delà des actuelles limites administratives.

S'il est un domaine qui est devenu méconnaissable en moins de deux générations, c'est bien celui du monde rural, abordé dans le chapitre 3 à travers neuf contributions qui en abordent des facettes parfois inattendues, où la composante humaine est souvent primordiale, y compris dans son évolution temporelle (voir par exemple le passage du " folklore " au " patrimoine rural " abordé d'emblée par le premier article). Le support de la réflexion peut être classiquement matériel (tel le patrimoine bâti du Perche) ou beaucoup plus complexe à appréhender (le cas du vignoble nantais est révélateur). Le rôle du monde associatif est justement mis en exergue à plusieurs reprises, mais il apparaît au fil des études présentées, que la nostalgie passéiste constitue bien souvent, sinon un écueil, du moins un haut-fond à l'égard duquel une certaine circonspection s'impose (à cet égard, une comparaison avec l'évolution des " fêtes folkloriques " qui firent naguère florès en Bretagne eut été intéressante). Quant à relativiser l'obsession actuelle du " tout économique "...

L'industrie est un autre domaine qui a beaucoup évolué dans la région, mais à partir de bases très diverses; il fut bien souvent tributaire de ressources ponc- 
tuelles, en matières premières (charbon, fer, étain, ardoise...) ou en énergie (cours d'eau, bois, charbon) et ces critères d'implantation, qui ne correspondent plus toujours à la structuration actuelle de l'espace, peuvent faire obstacle à une mise en valeur. Cependant, des épopées industrielles passionantes se sont déroulées hors de telles contingences (les articles de pêche, la mode, l'horlogerie, le textile). Pour ce dernier, les interférences révélées ici entre le "petit mouchoir rouge de Cholet ", l'histoire et le folklorisme sont particulièrement subtiles; quant à l'horlogerie de Tiffauges, elle fournit un cas emblématique d'antagonisme entre deux perceptions d'un même patrimoine, celle de son propriétaire légitime et celle d'une partie au moins de la collectivité qui s'en estime légataire.

Le dernier chapitre de cette partie centrale, "Nouvelles frontières du Patrimoine ", ne regroupe pas moins de treize études, la plupart relativement courtes mais très variées. Outre une évocation de la fabrique d'allumettes de Trélazé, transfuge du chapitre précédent, cette partie aborde aussi bien l'architecture hospitalière que l'archivage (de papier certes, mais aussi de témoignages oraux) en passant par le patrimoine scientifique et technique avant d'évoquer l'intrusion du Numérique dans l'exploitation des fonds documentaires comme des sites (cf. le cas du château de Tiffauges ou les illuminations quelque peu " déjantées " de la cathédrale du Mans).

Aussi riche et varié soit-il, le contenu de ce volume n'est pas exhaustif (l'aurait-il pu d'ailleurs?). Sans tomber dans le jeu facile consistant à épingler les manques, qu'il nous soit permis un léger étonnement devant l'absence de toute allusion à la construction navale nantaise et nazairienne, à la pétrochimie de basse-Loire ou au " commerce triangulaire ", domaines pourtant évoqués de façon allusive dans la préface du président de Région. Quant au souhait des directeurs de l'ouvrage dans leur introduction (que "Toute démarche patrimoniale [ne soit] pas seulement un repli frileux sur le passé mais aussi une projection dans le futur "), il est permis d'espérer que le travail considérable fixé sur ces pages contribuera grandement à lui donner chair. 